\title{
A single-dose live-attenuated vaccine prevents Zika virus pregnancy transmission and testis damage
}

\author{
Chao Shan', Antonio E. Muruato², Brett W. Jagger ${ }^{3}$, Justin Richner ${ }^{3}$, Bruno T.D. Nunes ${ }^{1,4}$, \\ Daniele B.A. Medeiros ${ }^{1,4}$, Xuping Xie ${ }^{1}$, Jannyce G.C. Nunes ${ }^{1,4}$, Kaitlyn M. Morabito ${ }^{5}$, Wing-Pui Kong ${ }^{5}$, \\ Theodore C. Pierson ${ }^{6}$, Alan D. Barrett 7,8,9,10, Scott C. Weaver 2,10,11,12, Shannan L. Rossi 7,8,9, \\ Pedro F.C. Vasconcelos ${ }^{4,13}$, Barney S. Graham ${ }^{6}$, Michael S. Diamond $3,14,15,16$ \& Pei-Yong Shi,10,11,13,17
}

Zika virus infection during pregnancy can cause congenital abnormities or fetal demise. The persistence of Zika virus in the male reproductive system poses a risk of sexual transmission. Here we demonstrate that live-attenuated Zika virus vaccine candidates containing deletions in the $3^{\prime}$ untranslated region of the Zika virus genome (ZIKV-3'UTR-LAV) prevent viral transmission during pregnancy and testis damage in mice, as well as infection of nonhuman primates. After a single-dose vaccination, pregnant mice challenged with Zika virus at embryonic day 6 and evaluated at embryonic day 13 show markedly diminished levels of viral RNA in maternal, placental, and fetal tissues. Vaccinated male mice challenged with Zika virus were protected against testis infection, injury, and oligospermia. A single immunization of rhesus macaques elicited a rapid and robust antibody response, conferring complete protection upon challenge. Furthermore, the ZIKV-3'UTR-LAV vaccine candidates have a desirable safety profile. These results suggest that further development of ZIKV-3' UTR-LAV is warranted for humans.

\footnotetext{
${ }^{1}$ Department of Biochemistry \& Molecular Biology, University of Texas Medical Branch, Galveston, TX 77555, USA. ${ }^{2}$ Department of Microbiology \& Immunology, University of Texas Medical Branch, Galveston, TX 77555, USA. ${ }^{3}$ Department of Medicine, Washington University School of Medicine, St. Louis, MO 63110, USA. ${ }^{4}$ Department of Arbovirology and Hemorrhagic Fevers, Evandro Chagas Institute, Ministry of Health, 67030-000 Ananindeua, Brazil. ${ }^{5}$ Vaccine Research Center, National Institute of Allergy and Infectious Diseases, National Institutes of Health, Bethesda, MD 20892, USA. ${ }^{6}$ Viral Pathogenesis Section, Laboratory of Viral Diseases, National Institute of Allergy and Infectious Disease, National Institutes of Health, Bethesda, MD 20892 , USA. ${ }^{7}$ Institute for Human Infections \& Immunity, University of Texas Medical Branch, Galveston, TX 77555, USA. ${ }^{8}$ Department of Pathology, University of Texas Medical Branch, Galveston, TX 77555, USA. ${ }^{9}$ Center for Biodefense \& Emerging Infectious Diseases, University of Texas Medical Branch, Galveston, TX 77555, USA. ${ }^{10}$ Sealy Center for Vaccine Development, University of Texas Medical Branch, Galveston, TX 77555, USA. ${ }^{11}$ Institute for Translational Science, University of Texas Medical Branch, Galveston, TX 77555, USA. ${ }^{12}$ Sealy Center for Structural Biology \& Molecular Biophysics, University of Texas Medical Branch, Galveston, TX 77555, USA. ${ }^{13}$ Department of Pathology, Pará State University, 67087-670 Belém, Brazil. ${ }^{14}$ Department of Molecular Microbiology, Washington University School of Medicine, St. Louis, MO 63110, USA. ${ }^{15}$ Department of Pathology \& Immunology, Washington University School of Medicine, St. Louis, MO 63110, USA. ${ }^{16}$ The Andrew M. and Jane M. Bursky Center for Human Immunology and Immunotherapy Programs, Washington University School of Medicine, St. Louis, MO 63110, USA. ${ }^{17}$ Department of Phamarcology \& Toxicology, University of Texas Medical Branch, Galveston, TX 77555, USA. Correspondence and requests for materials should be addressed to B.S.G. (email: bgraham@nih.gov) or to M.S.D. (email: diamond@wusm.wustl.edu) or to P.-Y.S. (email: peshi@utmb.edu)
} 
Z ika virus (ZIKV) belongs to the Flavivirus genus in the Flaviviridae family, and is closely related to other globally important flaviviruses, including dengue, yellow fever, Japanese encephalitis, West Nile, and tick-borne encephalitis viruses ${ }^{1}$. Flaviviruses have a single-stranded, positive-sense RNA genome consisting of a $5^{\prime}$ untranslated region (UTR), a single open reading frame (ORF), and a $3^{\prime}$ UTR. The single ORF encodes three structural (C-prM/M-E) and seven nonstructural (NS1-NS2A-NS2B-NS3-NS4A-NS4B-NS5) proteins. ZIKV is transmitted primarily by peridomestic Aedes mosquitoes, but also can be acquired through sexual, vertical, and blood transfusion routes $^{2,3}$. Although only about $20 \%$ of infected individuals develop clinical sign or symptoms, ZIKV infection in some adults is associated with the development of Guillain-Barré syndrome ${ }^{4}$. The most devastating manifestation of ZIKV infection is the array of congenital abnormalities in the fetuses and infants of women infected while pregnant, including microcephaly, craniofacial disproportion, spasticity, seizures, ocular abnormalities, cerebral calcification, and miscarriage $\mathrm{e}^{5,6}$. Longitudinal studies in humans in Brazil showed that among live infants born to symptomatic ZIKV-positive women, $42 \%$ had grossly abnormal clinical or brain imaging findings ${ }^{5}$. Furthermore, ZIKV infection can persist in the male reproductive tract, as infectious virus and viral RNA have been detected in semen up to 69 and 188 days after symptom onset, respectively ${ }^{7-9}$; infected males can transmit virus to sexual partners during this period of persistent infection ${ }^{10}$. Studies in mice have shown that ZIKV persistence in the testis causes damage to the seminiferous tubules, testicular atrophy, oligospermia, and reduced rates of fertility ${ }^{11-13}$. A number of promising ZIKV vaccine platforms have been developed, including inactivated, subunit (prM-E proteins expressed from DNA, RNA, or viral vectors), and live-attenuated vaccines ${ }^{14-21}$, several of which have entered phase I-II clinical trials 22,23 . Among these vaccine candidates, the prM-E RNA vaccine and live-attenuated vaccine (lacking NS1 glycosylations) were recently shown to protect against vertical transmission in a mouse pregnancy model ${ }^{24}$. In addition, the DNA vaccine expressing prM-E proteins was recently shown to protect mice against ZIKVinduced damages to testes ${ }^{25}$.

Here we show that live-attenuated ZIKV vaccine candidates containing deletions in the $3^{\prime} \mathrm{UTR}$ of the ZIKV genome (ZIKV-3' UTR-LAV) prevent viral transmission during pregnancy and testis damage in mice, as well as infection of nonhuman primates (NHPs). We also demonstrate a desirable safety profile of the vaccine candidates. Our data suggest that ZIKV-3'UTR-LAV merits further development for humans.

a

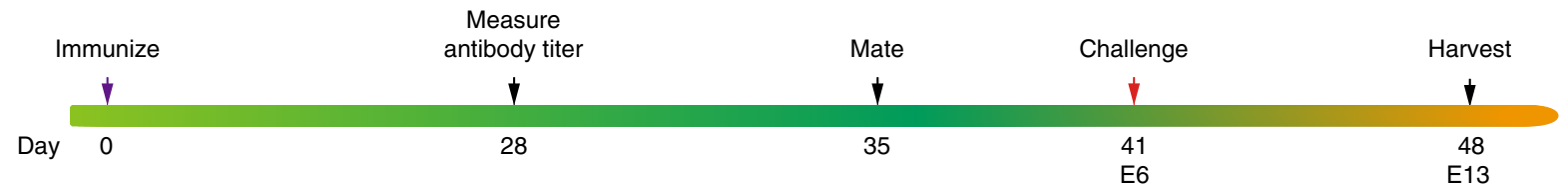

b

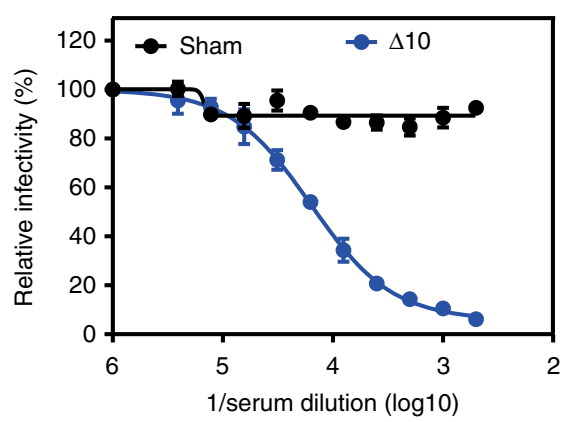

e

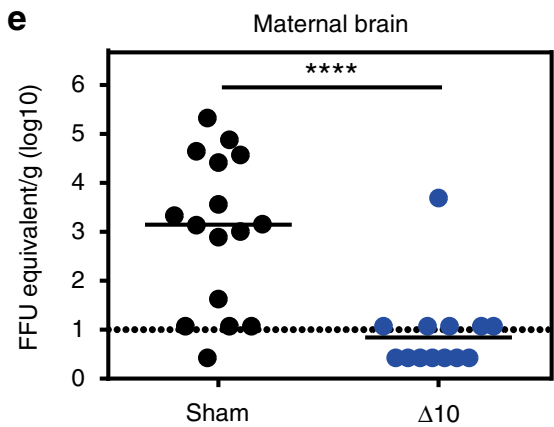

C

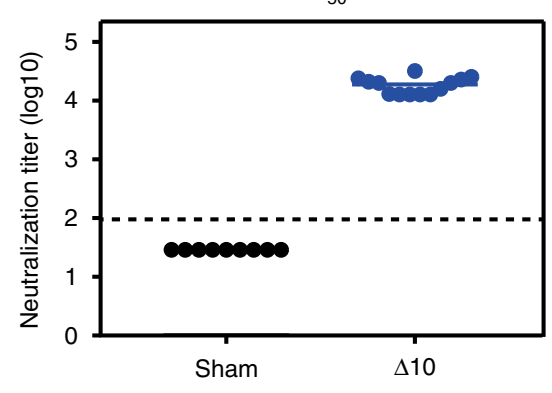

f

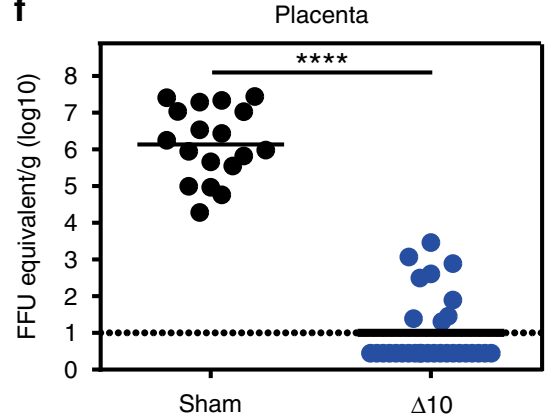

d

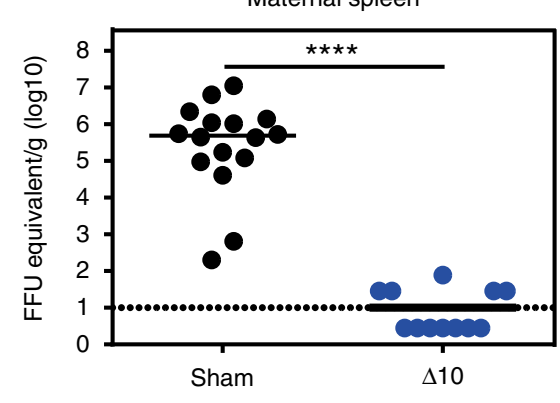

g

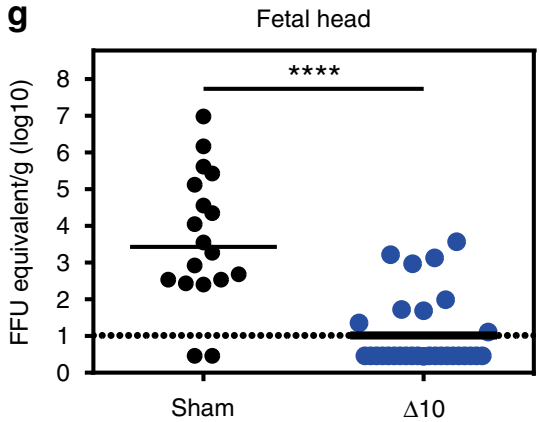

Fig. 1 ZIKV-3'UTR- $\triangle 10-L A V$ protects pregnant C57BL/6 mice and their developing fetuses. a Scheme of immunization of wild-type (WT) C57BL/6 female mice with $10^{5} \mathrm{FFU}$ of ZIKV-3'UTR- $\Delta 10$-LAV $(\Delta 10 ; n=12)$ or PBS sham $(n=16)$. b Serum was collected at day 28 post immunization and analyzed for neutralizing activity using an mCherry infectious ZIKV. Representative neutralization curves are shown. Error bars denote the SD of duplicate technical replicates. $\mathbf{c} \mathrm{NT}_{50}$ values of neutralizing antibodies were measured for individual animals. The dashed lines indicate the limit of detection (LOD) of the assay. d-g At day 35 post immunization, vaccinated female mice were mated with WT C57BL/6 males. A subset of the female mice developed vaginal plugs. Pregnant mice ( $n=8$ pooled from two independent experiments) were administered $2 \mathrm{mg}$ of anti-Ifnar1 blocking antibody on E5, and 1 day later (E6), challenged with $10^{5} \mathrm{FFU}$ of a pathogenic, mouse adapted ZIKV Dakar 41519 strain. On E13, animals were euthanized; maternal spleen (d), maternal brain (e), placenta (f), and fetal heads ( $(\mathbf{g})$ were harvested and quantified for viral RNA levels. Median viral RNA levels are indicated for each group. Asterisks indicate significant differences (Mann-Whitney test: ${ }^{\star \star \star \star} P$-value $<0.0001$ ). All negative samples are plotted at the half value of LOD. The results in the Figure are pooled from two independent experiments 
a

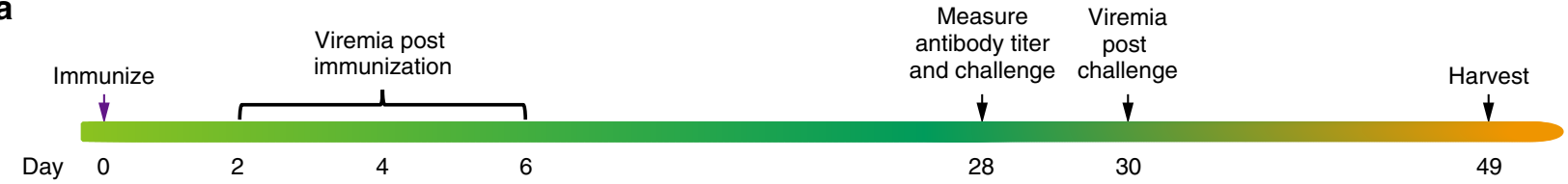

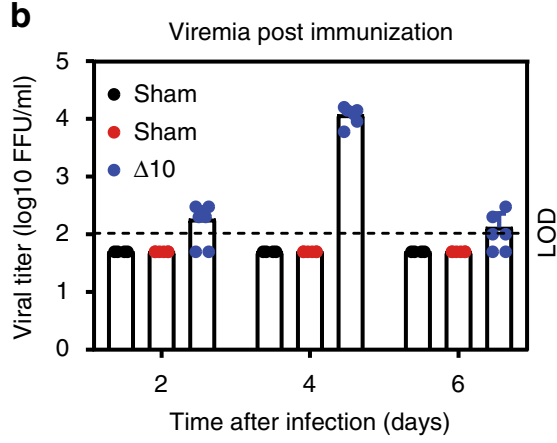

e

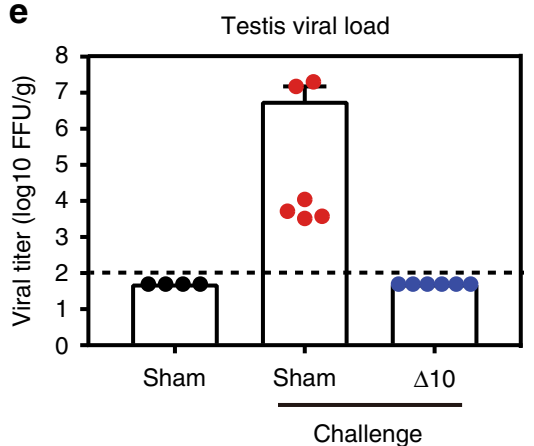

h

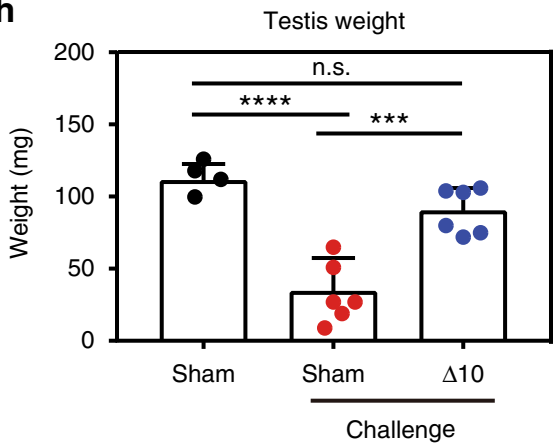

C

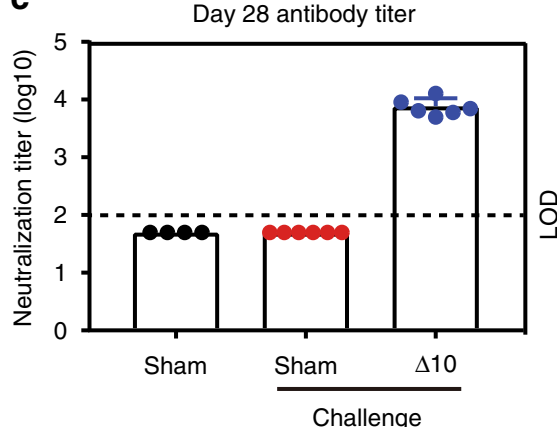

$\mathbf{f}$

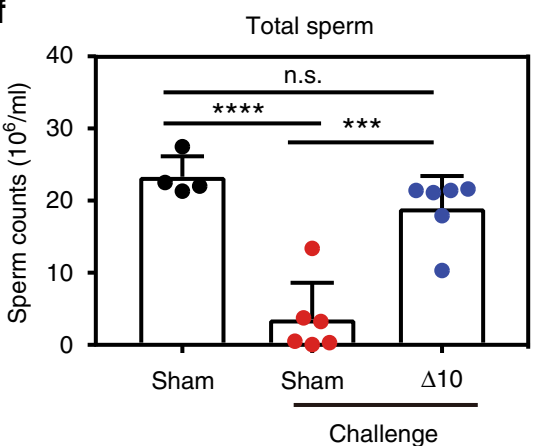

i d

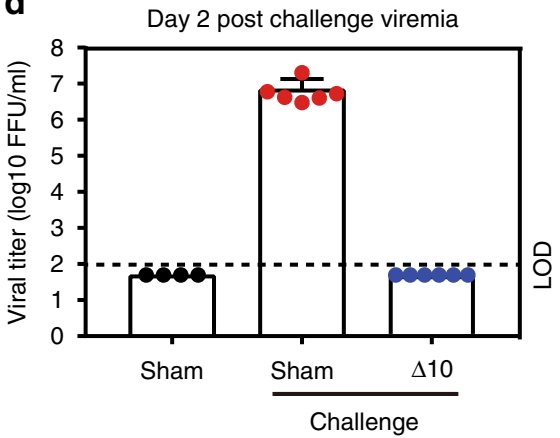

g

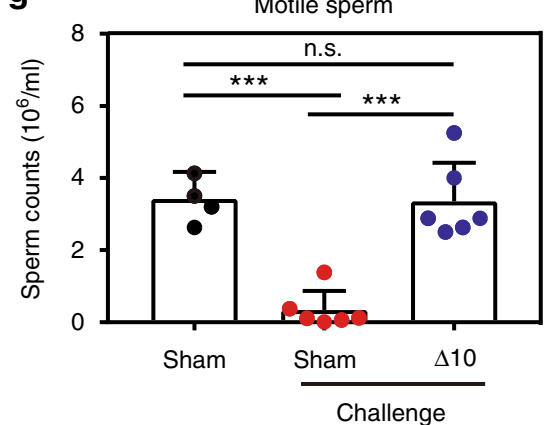

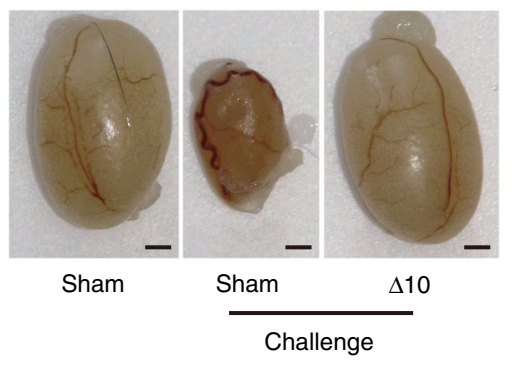

Fig. 2 ZIKV-3'UTR- $\Delta 10$-LAV protects young A129 male mice against testis infection and injury. a Scheme of immunization of 3-week-old A129 male mice with $10^{4} \mathrm{FFU}$ of ZIKV-3'UTR- $\Delta 10$-LAV $(\Delta 10 ; n=6)$ or PBS sham ( $n=4$ or 6 ). At day 28 post immunization, mice were measured for neutralization antibody titers. On the same day, mice from one sham group and mice from $\Delta 10$-immunized group were challenged with $10^{6} \mathrm{FFU}$ of ZIKV-PRVABC59, and viremia was measured at day 2 post challenge (day 30 post immunization). At day 49 post immunization, mice were analyzed for sperm counts and viral load in the testis. b Viremia after immunization with $\Delta 10$ vaccine candidate. ${ }^{N} T_{50}$ values of antibody neutralization at day 28 post immunization were measured for individual animals in each group. The dashed lines indicate the limit of detection (LOD) of the assay. d Viremia at day 2 post challenge (day 30 post immunization) with ZIKV PRVABC59. e Viral load in the testis at day 21 post challenge (day 49 post immunization). f, $\mathbf{g}$ Total (f) and motile (g) sperm counts at day 21 post challenge. h, $\mathbf{i}$ Testis weight (h) and representative images of testis (i) from animals from sham, sham with challenge, and $\Delta 10$-immunized and challenged groups at day 21 post challenge. Scale bar, $1 \mathrm{~mm}$. Asterisks indicate significant differences (one-way ANOVA: ${ }^{\star \star \star \star} P$-value $<0.0001 ;{ }^{\star \star \star} P$-value $<0.001$ ). Nonsignificant (n.s.), $P$-value $>0.05$. All negative samples are plotted at the half value of LOD. Error bars represent SDs

\section{Results}

Prevention of vertical transmission in pregnant mice. We recently developed a live-attenuated vaccine candidate containing a 10-nucleotide deletion in the $3^{\prime} \mathrm{UTR}$ of ZIKV genome (ZIKV-3'UTR- $\Delta 10-\mathrm{LAV}$ ); this vaccine conferred nearly sterilizing immunity after a single-dose immunization and showed an excellent safety profile in mice ${ }^{19}$. To test the ability of this vaccine candidate to prevent in utero transmission, we subcutaneously inoculated $10^{5}$ focus-forming units (FFU) of ZIKV-3'UTR- $\Delta 10$-LAV or Phosphate Buffered Saline (PBS- sham) into 8-week-old wild-type (WT) C57BL/6 female mice (Fig. 1a). Because mice are not a native host for ZIKV due in part to a species-dependent lack of antagonism of type I interferon (IFN) signaling 26,27 , we administered $0.5 \mathrm{mg}$ of anti-IFN alpha/ beta receptor 1 (anti-Ifnar1) blocking antibody to female mice 1 day prior to vaccination to facilitate transient replication of ZIKV-3'UTR- $\Delta 10-\mathrm{LAV}^{28}$. At day 28 post-vaccination, the animals were phlebotomized and serum was analyzed for neutralizing antibody (Fig. 1b); all ZIKV-3'UTR- 10 -LAVimmunized mice developed high neutralizing antibody titers of 
$18,900 \pm 5900($ mean $\pm \mathrm{SD} ; n=13)$, whereas, as expected, the PBS-immunized animals did not develop detectable neutralizing antibodies (Fig. 1c). At day 35 post vaccination, the immunized females were mated with 12-week-old WT C57BL/6 male mice and monitored for vaginal plugs (Fig. 1a).
At embryonic day 6 (E6), pregnant mice were challenged subcutaneously with $10^{5}$ FFU of a mouse-adapted, pathogenic ZIKV African strain Dakar $41519^{29}$; to facilitate ZIKV-Dakar dissemination to the maternal decidua and fetal placenta, the pregnant mice were administered $2 \mathrm{mg}$ of anti-Ifnarl antibody at
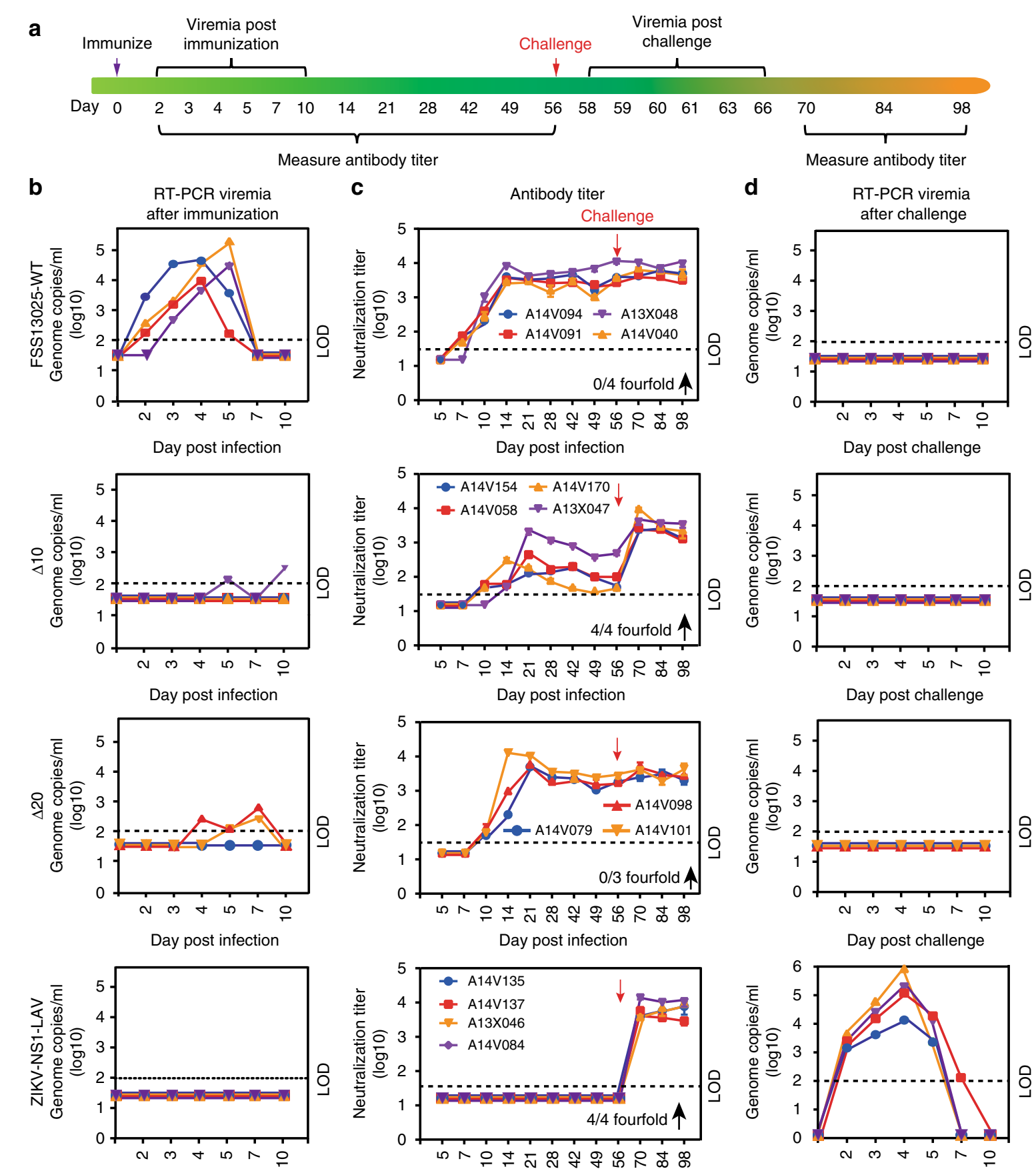

Day post infection
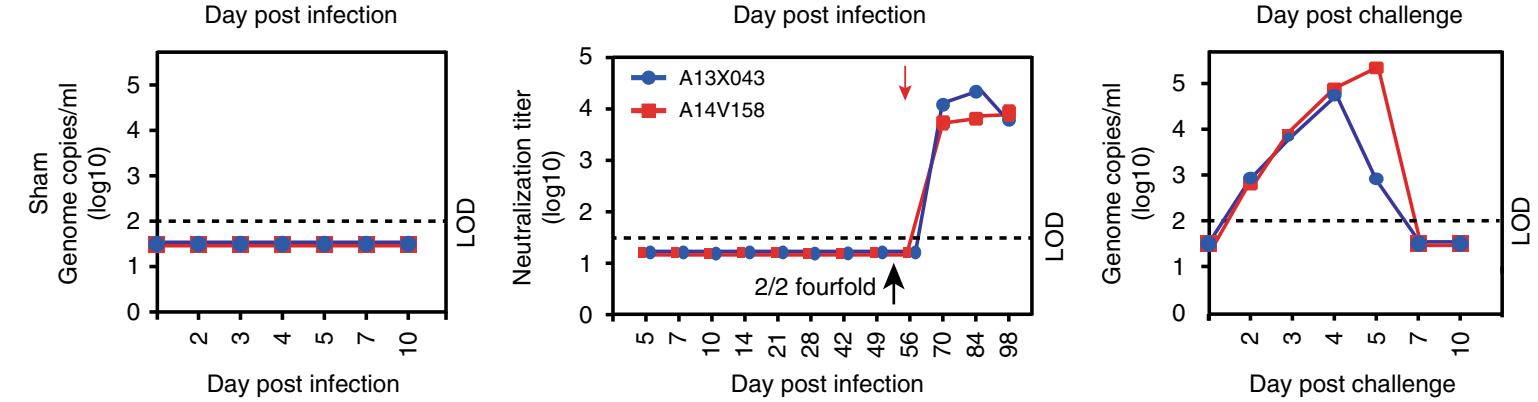
E5, 1 day before the challenge. At E13, maternal and fetal organs were harvested and measured for viral load.

After a single immunization, ZIKV-3'UTR- $\Delta 10-\mathrm{LAV}$ reduced median viral loads in the maternal spleen and brain by $\sim 49,000$-fold and $\sim 120$-fold, respectively (Fig. 1d, e). Placentas and fetal heads from the vaccinated dams showed 138,000-fold and 260 -fold decreases in viral RNA loads, respectively, when compared with the PBS-immunized dams (Fig. 1f, g). Notably, 21 of $30(70 \%)$ placentas and 21 of $30(70 \%)$ fetal heads from ZIKV-3'UTR- $\Delta 10$-LAV-immunized dams had viral RNA loads at or below the detection limit; no infectious virus was recovered by focus-forming assay from the placentas or fetal heads from the vaccinated dams (Supplementary Fig. 1a, b). In addition, the immune correlate between the $\mathrm{NT}_{50}$ values and the levels of infectious viruses detected in the placenta revealed an expected inverse relationship between neutralizing titers and levels of ZIKV particles in placenta (Supplementary Fig. 1c). Collectively, the data suggest that ZIKV-3'UTR- $\triangle 10-\mathrm{LAV}$ protects maternal organs from infection, prevents viral transmission to the fetus during an early stage of pregnancy, and limits fetal virus replication.

Protection of ZIKV-induced damages to testes. We examined the ability of ZIKV-3'UTR- $\Delta 10$-LAV to prevent testis infection and injury in Ifnar1 ${ }^{-/-}$A129 mice (Fig. 2a). Since male mice reach sexual maturity at 8 weeks of age, we tested the vaccine efficacy in two age groups of mice: 3-week-old young males; and 15-week-old adult males. Three-week-old young A129 male mice were vaccinated with a single-dose of $10^{4}$ FFU of ZIKV-3'UTR- $\Delta 10$-LAV or PBS sham. The ZIKV-3'UTR- $\Delta 10-\mathrm{LAV}$ generated a peak viremia of $1.2 \pm 0.34 \times 10^{4} \mathrm{FFU}(n=6)$ at day 4 post vaccination (Fig. $2 \mathrm{~b}$ ). At day 28 post vaccination, ZIKV-3'UTR- $\Delta 10$-LAV had induced robust neutralizing antibody titers of $7700 \pm 2600(n=6$; Fig. 2c). At the same day, the animals were challenged intraperitoneally with $10^{6} \mathrm{FFU}$ of an epidemic ZIKV strain from Puerto Rico (PRVABC59). No viremia was detected from the ZIKV-3'UTR- $\Delta$ 10-LAV-vaccinated mice after challenge, whereas the sham-vaccinated animals sustained a mean peak viremia of $7.1 \pm 5.9 \times 10^{6} \mathrm{FFU} / \mathrm{ml}(n=6)$ at day 2 post challenge (Fig. $\left.2 \mathrm{~d}\right)$. At day 21 post challenge, viral burden in the testis of PBS-immunized mice reached $5.8 \pm 8.4 \times 10^{6} \mathrm{FFU} / \mathrm{g}(n=6)$, whereas no infectious virus was detected in the testes of ZIKV-3'UTR- $\Delta 10$-LAV-immunized mice (Fig. 2e). Both total and motile sperm counts from the ZIKV-3'UTR- $\Delta 10$-LAV-immunized mice were equivalent to those from age-matched unvaccinated, unchallenged healthy male mice. In contrast, the PBS-immunized, ZIKV-challenged mice showed $85 \%$ and $90 \%$ reductions for total and motile sperm counts, respectively, at day 21 post infection (Fig. $2 \mathrm{f}$, g). Consistent with these data, the testis weight and size from the PBS-immunized, ZIKV-challenged mice were reduced, whereas no such reduction was observed in the ZIKV-3'UTR- $\Delta 10$-LAV-immunized, ZIKV-challenged animals (Fig. 2h, i).

In 15-week-old adult A129 male mice, vaccination with ZIKV-3'UTR- $\Delta 10$-LAV also protected against testis infection, injury, and oligospermia (Supplementary Fig. 2a-g). However, challenge of the PBS-vaccinated adult males with
ZIKV PRVABC59 did not reduce the testis weight and size (Supplementary Fig. 2h, i) as much as that observed in the corresponding sham-vaccinated, young males (Fig. 2h, i), suggesting an age-dependent testis pathology. Collectively, the results indicate that a single-dose immunization of ZIKV-3'UTR- $\Delta 10$-LAV protects the testis from infection and injury in male mice.

Efficacy in NHPs. To determine whether the efficacy in mice extends to NHPs, we evaluated the viremia, immunogenicity, and potency of ZIKV-3'UTR- $\Delta 10-\mathrm{LAV}$ in rhesus macaques (RMs; Fig. 3a). First, we assessed the level of attenuation of ZIKV-3'UTR- $\Delta 10$-LAV in RMs by comparing viremia to the parental WT virus. After subcutaneous inoculation with $10^{3} \mathrm{FFU}$, WT ZIKV (2010 Cambodian strain FSS 13025) produced high levels of viremia in each of the four inoculated RMs, with a mean peak viremia of $9.6 \times 10^{4}$ and $2.88 \times 10^{5}$ genome copies $/ \mathrm{ml}$ at days 4 and 5 post infection, respectively (Fig. 3b, top panel). In contrast, only one of the four ZIKV-3'UTR- $\Delta 10$-LAV-inoculated RMs exhibited viremia, and this level was just above the limit of detection of our quantitative reverse transcriptase-PCR (qRT-PCR) assay (Fig. 3b, second panel); thus, ZIKV-3'UTR$\triangle 10$-LAV is highly attenuated in RMs. We next evaluated the immunogenicity of ZIKV-3'UTR- $\Delta 10$-LAV by measuring neutralizing antibodies from serum at days 5-98 post immunization. Neutralizing antibodies elicited by WT ZIKV were detectable at day $7-10$, peaked at $1 / 1000-1 / 10,000$ at day 14 , and plateaued thereafter (Fig. 3c, top panel). Compared with the WT ZIKV infection, ZIKV-3'UTR- $\Delta 10-\mathrm{LAV}$-inoculated animals showed slightly delayed production and lower levels of neutralizing antibody, with titers of $\sim 1 / 100$ at days $21-56$ (Fig. 3c, second panel). At day 56 post immunization, all RMs were challenged with $10^{3} \mathrm{FFU}$ of the epidemic ZIKV strain PRVABC59. Notably, no viremia was detected upon challenge in any of the RMs that were pre-infected with WT ZIKV or vaccinated with the ZIKV-3'UTR- $\Delta 10-\mathrm{LAV}$ (Fig. 3d, top two panels). As controls, two PBS-inoculated RMs were challenged in parallel and high levels of viremia were measured (Fig. 3d, bottom panel). In addition to analysis of viral RNA by qRT-PCR, we also performed focus-forming assays to measure infectious virus; infectious ZIKV was detected only in naive RMs challenged with WT ZIKV (Supplementary Fig. 3).

To examine whether the ZIKV challenge resulted in boosted immune responses, we measured neutralizing activity post challenge. No increase in neutralizing activity was observed in the WT ZIKV-vaccinated RMs after challenge (Fig. 3c, top panel), indicating that the initial infection likely conferred sterilizing immunity. In contrast, the neutralizing antibody titers rose after challenge from $\sim 1 / 100$ to $1 / 1000-1 / 10,000$ in the ZIKV-3'UTR- $\Delta 10$-LAV-immunized animals (Fig. 3c, second panel), demonstrating an anamnestic response and suggesting a low level of infection after challenge that was not detectable by qRT-PCR of serum. As expected, the PBS-inoculated control RMs increased their neutralizing titers to $\sim 1 / 10,000$ after challenge (Fig. 3c, bottom panel).

Fig. 3 ZIKV-3'UTR- $\triangle 10-L A V$ and ZIKV-3'UTR- $\Delta 20-L A V$ protect rhesus macaques (RMs) from ZIKV infection. a Scheme of immunization of RMs with $10^{3}$ FFU of WT ZIKV strain FSS13025 $(n=4)$, ZIKV-3'UTR- $\Delta 10-$ LAV $(\Delta 10 ; n=4)$, ZIKV-3'UTR- $\Delta 20-$ LAV $(\Delta 20 ; n=3)$, ZIKV-NS1-LAV or PBS sham $(n=2)$ via the subcutaneous route. $\mathbf{b}$ Viremia was measured at days $2,3,4,5,7$, and 10 post immunization by qRT-PCR. Each colored line represents data from a different animal in each group. The dashed line indicates the limit of detection (LOD) of the assay. c Pre- and post-challenge antibody neutralization titers. On various days post immunization, sera were measured for neutralizing titers using an mCherry ZIKV infection assay. Red arrows indicate challenge with $10^{3} \mathrm{FFU}$ of epidemic ZIKV strain PRVABC59 via the subcutaneous route at day 56 post immunization. The number of animals whose antibody neutralization titers increased by $\geq 4$-fold after challenge is indicated by symbol " $\uparrow$ " for each experimental group. d Post-challenge viremia. Viremia was measured by qRT-PCR at days 2, 3, 4, 5, 7, and 10 post challenge. All negative samples are plotted at the half value of LOD. Error bars represent SDs 
a

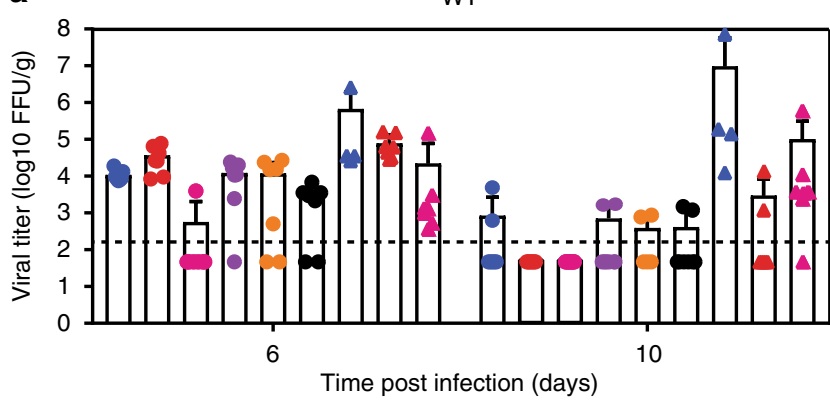

b
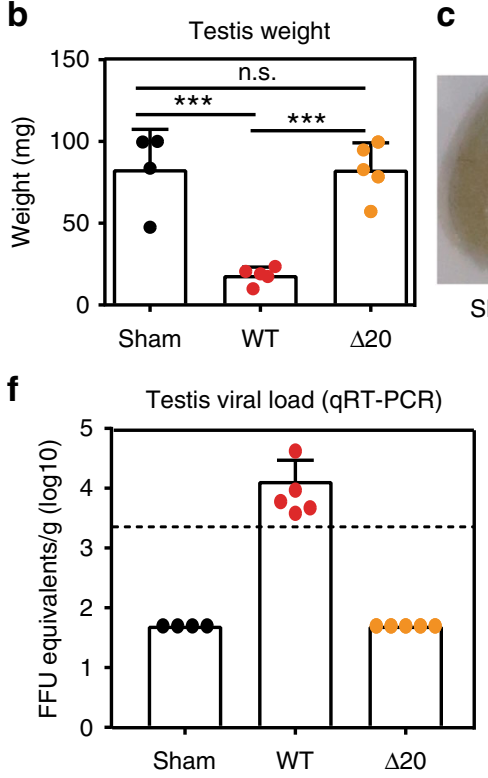

C

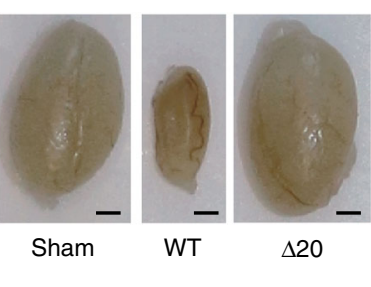

g

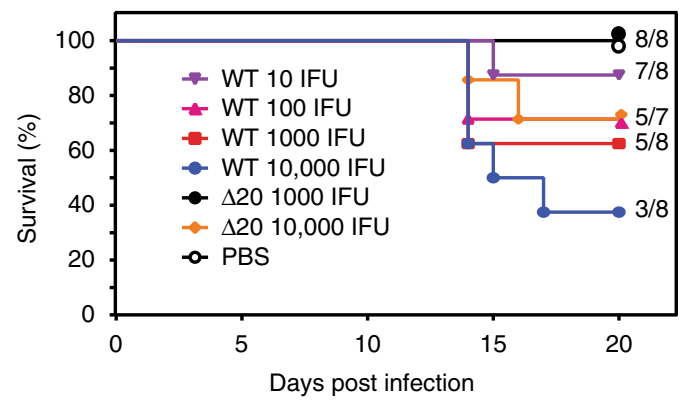

d

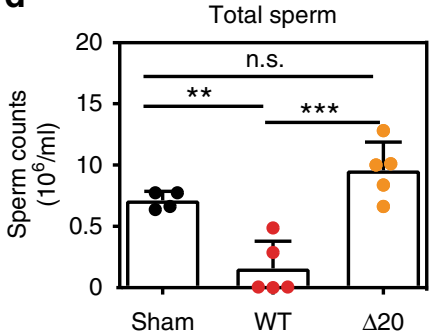

$\Delta 20$
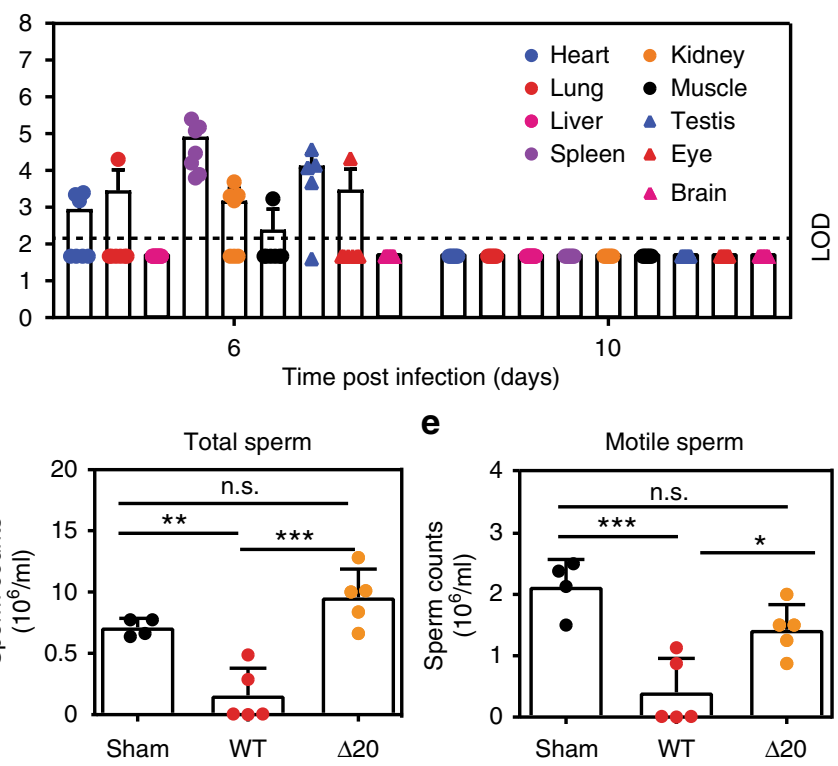

h

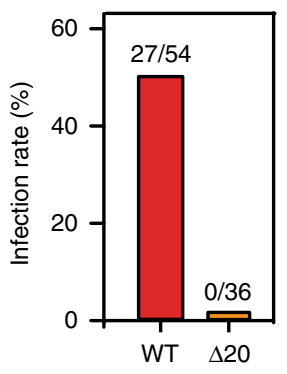

Fig. 4 Safety evaluation of ZIKV-3'UTR- $\Delta 20-L A V(\Delta 20)$ vaccine candidate. a Viral loads in organs of infected A129 mice. Three-week-old A129 mice $(n=7)$ were subcutaneously immunized with $10^{3} \mathrm{FFU}$ of WT ZIKV FSS13025 (left panel) and its derivative $\Delta 20$ vaccine candidate (right panel).

Organs from infected mice were collected and homogenized at days 6 and 10 post infection. The amounts of viruses were quantified on Vero cells using a focus-forming assay. The mean results from seven animals are presented. Bars denote standard errors. The dashed lines indicate the limit of detection (LOD) of the assay. b-f Effect of $\Delta 20$ vaccination on the testis. Three-week-old A129 mice $(n=5)$ were subcutaneously infected with $1 \times 10^{3} \mathrm{FFU}$ of WT ZIKV FSS13025 or $\Delta 20$ vaccine candidate. At day 28 post infection, animals from each group were analyzed for testis weight (b), testis size (c), total sperm counts (d), motile sperm counts (e), and viral RNA load (f). Scale bar, $1 \mathrm{~mm}$. $\mathbf{g}$ Comparison of neurovirulence of WT ZIKV FSS13025 and $\Delta 20$ vaccine candidate in outbred CD-1 mice. One-day-old CD-1 mice ( $n=7-8 /$ group) were injected intracranially with $10-10^{4} \mathrm{FFU}$ of WT ZIKV or $10^{3}-10^{4} \mathrm{FFU}$ of $\Delta 20$ vaccine candidate. Surviving mice and total infected animals are indicated. $\mathbf{h}$ Analysis of vector competency. Aedes aegypti were fed on artificial blood-meals spiked with $10^{6} \mathrm{FFU} / \mathrm{ml}$ of WT ZIKV FSS13025 or $\Delta 20$ vaccine virus. At day 7 post feeding, individual engorged mosquitoes were assayed for infection by immunostaining of viral protein expression on inoculated Vero cells. The number of infected mosquitos and total number of engorged mosquitoes are indicated. Asterisks indicate significant differences (one-way ANOVA: ${ }^{\star \star \star} P$-value $<0.001 ;{ }^{\star \star} P$-value $<0.01 ;{ }^{\star} P$-value $<0.05$ ). Nonsignificant (n.s.) with $P$-value $>0.05$. All negative samples are plotted at the half value of LOD. Error bars represent SDs

Since ZIKV-3'UTR- $\Delta$ 10-LAV did not elicit sterilizing immunity in RMs, we evaluated whether a second live-attenuated vaccine candidate $Z I K V-3^{\prime} \mathrm{UTR}-\Delta 20-\mathrm{LAV}^{19}$, which contains a 20 -nucleotide deletion in the $3^{\prime} \mathrm{UTR}$, could induce a stronger immune response. ZIKV-3'UTR- $\Delta 20$-LAV was shown previously, and paradoxically, to be less attenuated than ZIKV$3^{\prime}$ UTR- $\triangle 10$-LAV in A129 mice, most likely because it is less sensitive to type I IFN inhibition compared to ZIKV-3'UTR- $\Delta 10$ $\mathrm{LAV}^{19}$. After subcutaneous inoculation of $10^{3} \mathrm{FFU}$ of ZIKV-3' UTR- $\Delta 20-\mathrm{LAV}$, two of the three RMs had low, but detectable viremia (Fig. 3b, third panel). The immunized animals rapidly produced neutralizing antibodies by day 10 , with inhibitory titers plateauing at $1 / 1000-1 / 10,000$ by days $14-21$ (Fig. 3c, third panel). After challenge with $10^{3}$ FFU of ZIKV PRVABC59 at day 56 , viremia was not detected by qRT-PCR (Fig. 3d, third panel) and no rise in neutralizing antibody titers was observed (Fig. 3c, third panel) in the ZIKV-3'UTR- $\Delta 20-\mathrm{LAV}$-immunized animals. Although low number of animals were used for each vaccine candidate, the results suggest that a single-dose vaccination of ZIKV-3'UTR- $\triangle 20$-LAV induces sterilizing immunity in NHPs (i.e., no detectable viremia and no increase of neutralizing antibody titer after challenge).

We also evaluated another live-attenuated ZIKV vaccine candidate encoding an NS1 without glycosylation (ZIKV-NS1LAV) in RMs. ZIKV-NS1-LAV was recently shown to prevent in utero transmission in a mouse pregnancy model ${ }^{24}$. After subcutaneous immunization of four RMs with $10^{3} \mathrm{FFU}$ of ZIKVNS1-LAV, none of the animals showed any detectable viral RNA (Fig. 3b, fourth panel). Back titering of the ZIKV-NS1-LAV inoculum using a focus-forming assay confirmed the infectivity of viral stock with the expected infectious titer. Unexpectedly, the immunization did not ellicit any neutralizing activity 
(Fig. 3c, fourth panel). After challenge with $10^{3} \mathrm{FFU}$ of ZIKV PRVABC59 at day 56, all four animals displayed robust viremia (Fig. 3d, fourth panel) and generated neutralizing antibody titers (Fig. 3c, fourth panel). The results indicate that ZIKV-NS1-LAV is incapable of replicating and triggering antibody response in RMs.

Testis protection and safety analysis of ZIKV-3'UTR- $\Delta 20$ LAV. Because of the highly desirable sterilizing immunity induced by ZIKV-3'UTR- $\triangle 20-\mathrm{LAV}$ in RMs, we further tested its efficacy and safety. Similar to ZIKV-3'UTR- $\Delta 10$-LAV, immunization of male A129 mice with $10^{3}$ FFU of ZIKV-3'UTR- $\Delta 20$ LAV completely prevented viral infection and testis injury after challenge with ZIKV PRVABC59, as determined by a lack of detectable viremia post challenge, the absence of oligospermia, and no decrease in testis weight and size (Supplementary Fig. 4). Next, five sets of experiments were performed to characterize the safety of ZIKV-3'UTR- $\Delta 20$-LAV. First, we measured the organ viral loads after subcutaneous inoculation of A129 mice with $10^{3}$ FFU of ZIKV-3'UTR- $\triangle 20$-LAV or parental WT ZIKV (Fig. 4a). At day 6 post infection, WT ZIKV-infected mice exhibited high viral loads in all tested organs, whereas no infectious virus was detected $\left(\leq 10^{2} \mathrm{FFU} / \mathrm{ml}\right)$ in liver or brain from the ZIKV-3'UTR$\Delta 20$-LAV-infected mice, with other organs (except spleen) exhibiting lower levels of the vaccine virus than those of the WTinfected animals. At day 10 post infection, WT ZIKV-infected mice retained viral loads in the heart, spleen, kidney, muscle, testis, eye, and brain, whereas no organs from the ZIKV-3'UTR$\triangle 20$-LAV-infected mice had any detectable virus. Second, we examined the potential adverse effect of ZIKV-3'UTR- $\Delta 20-\mathrm{LAV}$ on the testis in 3-week-old A129 mice. As expected, at day 21 post infection, WT ZIKV infection reduced testis weight and size (Fig. 4b, c), lowered total and motile sperm counts (Fig. 4d, e), and resulted in viral RNA in the shrunken testis (Fig. 4f). In contrast, ZIKV-3'UTR- $\Delta 20$-LAV did not affect sperm counts or testis weight and size (Fig. 4b-e), with no detectable viral RNA in the testes (Fig. 4f). Third, we evaluated the neurovirulence of ZIKV-3'UTR- $\triangle 20$-LAV through intracranial inoculation of 1day-old outbred, immunocompetent CD-1 mice (Fig. 4g). As reported previously ${ }^{19}$, neonates succumbed to WT ZIKV infection; even a dose of only $10 \mathrm{FFU}$ resulted in $13 \%$ mortality (Fig. $4 \mathrm{~g}$ ). In contrast, no mortality was observed in mice that were inoculated with $10^{3} \mathrm{FFU}$ of ZIKV-3'UTR- $\triangle 20$-LAV; however, infection with $10^{4} \mathrm{FFU}$ of ZIKV-3'UTR- $\Delta 20$-LAV resulted in a mortality rate of $29 \%$. Fourth, we tested if the vaccine candidate could infect Aedes aegypti mosquitoes, the main vector of $\mathrm{ZIKV}^{30}$, 31 . After feeding on artificial blood-meals containing $10^{6} \mathrm{FFU} / \mathrm{ml}$ of ZIKV-3'UTR- $\triangle 20$-LAV or WT ZIKV, $50 \%$ of the engorged mosquitoes were infected by WT ZIKV, whereas no mosquitoes were infected by ZIKV-3'UTR- $\Delta 20$-LAV (Fig. 4h). Finally, we tested the stability of ZIKV-3'UTR- $\Delta 20-\mathrm{LAV}$ in cell culture. After continuous culture of ZIKV-3'UTR- $\Delta 20$-LAV on Vero cells (an approved cell line for vaccine production ${ }^{32}$ ) for five rounds, all recovered P5 viruses (derived from three independent experiments) retained the 20-nucleotide deletion. However, the P5 viruses accumulated additional mutations in the E- and NS1encoding genes (Supplementary Table 1), which may represent Vero-cell-adaptive mutation(s) or compensatory mutation(s) to 3'UTR deletion. Further passaging of the viruses to P10 did not change the 20-nucleotide deletion, indicating that the deletion is stable in cell culture. Moreover, we passaged ZIKV-3'UTR- $\Delta 20$ LAV in A129 mice for three rounds (3 days/round); all recovered viruses retained the 20 -nucleotide deletion, further suggesting the stability of the mutant virus. Taken together, these results demonstrate an excellent safety profile of ZIKV-3'UTR- $\Delta 20$ -
LAV, including limited, transient viral loads in mouse organs, no adverse effect on testicular function, decreased neurovirulence, incompetency to infect mosquitoes, and good stability.

\section{Discussion}

Our results showed that a single immunization of ZIKV-3'UTR$\triangle 10$-LAV prevented maternal-to-fetal transmission early during pregnancy in C57BL/6 mice. Although no infectious challenge virus was detected, very low levels of viral RNA were recovered from $\sim 30 \%$ of placentas and fetal heads from the vaccinated dams after challenge; these breakthrough viral RNAs might derive from stable antibody-virus complexes, which can last for several days in vivo ${ }^{33}$. The clinical implications of such breakthrough noninfectious viral RNA remain to be determined. In male A129 mice, a single-dose immunization of either ZIKV-3'UTR- $\Delta 10$ LAV or ZIKV-3'UTR- $\triangle 20-\mathrm{LAV}$ prevented testis infection and injury after challenge, indicating an additional benefit of vaccination to protect the male reproductive system. Notably, unprotected young A129 mice (3 or 7 weeks old when infected) infected with ZIKV developed smaller testes, whereas adult mice (19 weeks old when infected) did not, suggesting that ZIKV infection might cause more severe reproductive damage in younger males. The clinical relevance of this observation remains to be verified in NHPs and in humans. In NHPs, a single-dose vaccination with ZIKV-3'UTR- $\Delta 10-\mathrm{LAV}$ or ZIKV-3'UTR- $\Delta 20$ LAV induced sufficient immune responses to prevent viremia, with the ZIKV-3'UTR- $\Delta 20$-LAV eliciting greater immunogenicity, as reflected by its ability to induce sterilizing immunity against challenge. One limitation of the current NHP results is the low number of animals used for each vaccine candidates $(n=$ 3-4). ZIKV vaccine-induced sterilizing immunity might be critical for protection against congenital abnormalities in humans.

Live-attenuated vaccines generally have the advantage of single dose, rapid induction of durable immunity. Since ZIKV is endemic primarily in low-income countries, a vaccine with single-dose efficacy is of practical importance, particularly when controlling an explosive outbreak or immunizing populations in remote areas where multiple doses and periodic boosting will be challenging ${ }^{34}$. Thus, live-attenuated vaccines may be useful for immunizing populations living in and traveling to ZIKV-endemic areas. Besides our ZIKV-3'UTR-LAV, a single-dose immunization with nucleoside-modified mRNA expressing ZIKV prM-E $(50 \mu \mathrm{g})^{18}$ or a recombinant rhesus adenovirus serotype 52 vector expressing ZIKV prM-E (10 ${ }^{11}$ viral particles $)^{16}$ was also shown to rapidly elicit antibody response and prevent viremia in NHPs; whether these two vaccines achieved sterilizing immunity was not determined. All other vaccine platforms, including inactivated vaccine and prM-E DNA vaccine, need two immunizations to ellicit robust antibody response for viremia protection in NHPs ${ }^{15}$, 16. It is conceivable that in immunocompromised individuals and pregnant women, vaccination with live-attenuated virus may be contraindicated to avoid potential adverse risks. These individuals could be protected using inactivated, subunit, or genebased replication-defective vaccines. Therefore, there is a need to develop multiple vaccine platforms in parallel to provide complementary options for preventing and controlling ZIKV infection and disease.

\section{Methods}

Experimental systems and measurements. Various in vivo systems were employed to characterize the safety and efficacy of vaccine candidates, including C57BL/6J mouse pregnancy, A129 mouse testis protection, viral loading in A129 mouse organ, CD-1 mouse neurovirulence, and RMs efficacy (see details below) Since the protocols for each of these experimental systems have been previously established (e.g., inoculum dose, infection route, challenge dose, and end-point measurement), we did not attempt to change these optimized parameters when testing our vaccine candidates. Therefore, different inoculum doses, challenge 
doses, and end-point measurements (e.g., qRT-PCR to measure viral RNA and focus-forming assay to measure infection virus) were used in different in vivo systems according to the established protocols. The detailed information is described below and appropriately indicated in Results.

Ethics statement. Mouse studies were performed in accordance with the recommendations in the Guide for the Care and Use of Laboratory Animals of the National Institutes of Health. The protocols were approved by the Institutional Animal Care and Use Committee (IACUC) at the Washington University School of Medicine (Assurance Number A3381-01) and the IACUC at the University of Texas Medical Branch (UTMB; Protocol Number 0209068B). Dissections and footpad injections were performed under anesthesia that was induced and maintained with ketamine hydrochloride and xylazine at the Washington University or isoflurane at UTMB. All efforts were made to minimize animal suffering. RMs experiments were reviewed and approved by the Vaccine Research Center Animal Care and Use Committee at the National Institute of Allergy and Infectious Diseases, the National Institutes of Health. The NHP experiments were performed in compliance with the pertinent regulations and policies from the National Institutes of Health.

Viruses and cells. The ZIKV Cambodian strain FSS13025 (GenBank number KU955593.1) was produced from an infectious cDNA clone ${ }^{35}$. The ZIKV-3'UTR$\triangle 10-\mathrm{LAV}$ and ZIKV-3'UTR- $\Delta 20$-LAV strains were generated as described. The Zika Puerto Rico strain PRVABC59 (GenBank number KU501215) and Dakar 41519 strain (GenBank number HQ234501.1) were obtained originally from Dr Robert Tesh from the World Reference Center of Emerging Virus and Arboviruses (WRCEVA) at UTMB. The mouse-adapted ZIKV-Dakar 41519 strain was passaged twice in $\mathrm{Ragl}^{-1-}$ mice (Jackson Laboratories) and as described previously ${ }^{29}$. Vero cells were purchased from the American Type Culture Collection (CCL-81; Bethesda, MD, USA), and maintained at $37^{\circ} \mathrm{C}$ with $5 \% \mathrm{CO}_{2}$ in a high glucose Dulbecco modified Eagle medium (DMEM; Invitrogen, Carlsbad, CA, USA) with $10 \%$ fetal bovine serum (HyClone Laboratories, Logan, UT, USA) and $1 \%$ penicillin/streptomycin (Invitrogen). All cell lines tested negative for mycoplasma.

Antibodies. The following antibodies were used in this study: anti-mouse Ifnar 1 monoclonal antibody (clone MAR1-5A3; Leinco Technologies, Inc., St. Louis, MO, USA); ZIKV-specific hyper-immune ascites fluids (obtained from WRCEVA); antimouse IgG antibody labeled with horseradish peroxidase (KPL, Gaithersburg, MD, USA); and goat anti-mouse IgG conjugated with Alexa Fluor 488 (Thermo Fisher Scientific, Providence, RI, USA).

A129 mouse experiment. A129 mice were bred in the animal facilities at UTMB. All mice were housed in pathogen-free mouse facilities. Three-week- or 15-weekold male A129 mice were infected with PBS (two sham groups), $10^{4} \mathrm{FFU}$ of ZIKV$3^{\prime}$ UTR- $\Delta 10$-LAV $(\Delta 10)$, or $10^{3}$ FFU of ZIKV-3'UTR- $\Delta 20$-LAV $(\Delta 20)$. Mice were anesthetized and bled via the retro orbital sinus for viremia testing. At day 28 post immunization, mice were measured for neutralizing antibody titers using an mCherry ZIKV infection assay ${ }^{19}$. On the same day, one sham group of PBSimmunized mice and $\Delta 10$ - and $\Delta 20$-immunized mice were challenge with $10^{6} \mathrm{FFU}$ of ZIKV PRVABC59. Another sham group of mice was used as an unchallenged negative control. At day 49 post immunization, mice were euthanized and necropsied. Epididymis and testes were harvested immediately as previously described $^{36}$. Motile and nonmotile sperms were counted manually on a hemocytometer by microscopy. Total sperm counts equal to the sum of motile and nonmotile sperms. For quantification of viral loads, testes were homogenized and infectious viral levels were measured by a focus forming assay or qRT-PCR ${ }^{19}$. The qRT-PCT primer/probe set includes forward primer (1193F: $5^{\prime}$ -

CCGCTGCCCAACACAAG-3'), reverse primer (1269R: 5'-CCAC

TAACGTTCTTTTGCAGACAT- $3^{\prime}$ ), and probe (5'-FAM/AGCCTACCT/ZEN/

TGACAAGCAATCAGACACTCAA/3IABkFQ- $\left.3^{\prime}\right)$. The probe contains a $5^{\prime}$-FAM reporter dye, $3^{\prime}$ IBFQ quencher, and an internal ZEN quencher.

Mouse pregnancy experiment. C57BL/6J mice were bred and housed in pathogen-free mouse facilities at Washington University School of Medicine. One day prior to immunization, the 8-week-old female C57BL/6J mice were dosed with $0.5 \mathrm{mg}$ of anti-Ifnarl antibody via an intraperitoneal route. Subsequently, mice were subcutaneously inoculated in the footpad with $10^{5}$ FFU of ZIKV-3'UTR- $\Delta 10$ LAV or PBS sham. Immunized WT C57BL/6 female mice were mated with naive WT male mice. At E5, pregnant dams were injected intraperitoneally with $2 \mathrm{mg}$ of anti-Ifnar1 antibody. On E6, mice were inoculated subcutaneously with $10^{5} \mathrm{FFU}$ of mouse-adapted ZIKV Dakar 41519 via footpad injection. All animals were sacrificed on E13 and analyzed for viral loads in placentas, fetuses, and maternal tissues. Briefly, maternal blood, organs from dams (brain and spleen), and fetuses (placenta and fetal head) were collected. Serum was prepared after coagulation and centrifugation. Organs were weighed and homogenized using a bead-beater apparatus (MagNA Lyser, Roche). Viral RNA was extracted from serum and tissue samples using the RNeasy Mini kit (Qiagen). The viral RNA levels were determined by
TaqMan one-step qRT-PCR on an ABI 7500 Fast Instrument using standard cycling conditions. Viral burden is expressed on a log10 scale as viral RNA equivalents per gram or per milliliter after comparison with a standard curve produced using serial fivefold dilutions of ZIKV RNA from known quantities of infectious virus. The following primer/probe set was used for ZIKV qRT-PCR: forward primer (1183F: 5'-CCACCAATGTTCTCTTGCAGACATATTG-3'), reverse primer (1268R: 5'-TTCGGACAGCCGTTGTCCAACACAAG-3'), and probe (1213F: 5'-56-FAM/AGCCTACCT TGACAAGCAGTC/3IABkFQ-3'). Wherever indicated, viral burden for some samples was determined by focusforming assay on Vero cells ${ }^{20}$.

Quantification of viral load in organs from A129 mice. A129 mice were infected and organs were quantified for viral load using a focus-forming assay ${ }^{20}$. Viral RNA in testis also was quantified by qRT-PCR. Briefly, testes were harvested and placed in DMEM with beads for homogenization. After homogenization, the supernatant was used to extract viral RNA using RNeasy Mini kit (Qiagen). Extracted RNA was eluted in $40 \mu \mathrm{l}$ RNase-free water. qRT-PCR assays were performed on the LightCycler 480 System (Roche) following the manufacturer's protocol by using a $50 \mu \mathrm{l}$ reaction of the QuantiTect Probe RT-PCR Kit (Qiagen) and $10 \mu \mathrm{l}$ RNA template. The viral load was calculated based on a standard curve produced using serial 10 fold dilutions of ZIKV RNA from known quantities of infectious virus. The qRTPCT primer/probe set includes forward primer (1193F: 5'-CCGCTGCCCAACACAAG-3'), reverse primer (1269R: 5' -CCACTAACGTTCTTTTGCAGACAT-3'), and probe (5'-FAM/AGCCTACCT/ZEN/TGACAAGCAATCAGACACTCAA/ $3 \mathrm{IABkFQ}-3^{\prime}$ ). The probe contains a $5^{\prime}$-FAM reporter dye, $3^{\prime}$ IBFQ quencher, and an internal ZEN quencher.

Vaccination of NHPs. RM (Macaca mulatta) experiments were performed at Bioqual, Inc. (Rockville, MD, USA). All animal experiments were reviewed and approved by the Animal Care and Use Committee of the Vaccine Research Center, the National Institute of Allergy and Infectious Diseases, the National Institutes of Health. Animals were housed and cared in accordance with local, state, federal, and institutional policies in an American Association for Accreditation of Laboratory Animal Care-accredited facility at the Bioqual Inc. RMs (3-4/group) were randomized by body weight, gender, and age, and subcutaneously administered with $10^{3}$ FFU of parental WT ZIKV strain FSS13025, ZIKV-3'UTR- 10 -LAV, ZIKV-3' UTR- $\triangle 20$-LAV, or PBS sham at day 0 . Blood was collected at days $2,3,4,5,7$, and 10 for viremia testing and weekly for analysis of antibody responses by an mCherry ZIKV neutralization assay ${ }^{19,37}$. The immunized animals were subcutaneously challenged with $10^{3} \mathrm{FFU}$ of ZIKV strain PRVABC59 at week 8. Blood samples were collected for determination of viral load at days $2,3,4,5,7$, and 10 , and neutralizing antibody at weeks 2,4 , and 6 post challenge.

Viremia from RMs was quantified by a qRT-PCR assay and focus-forming assay as described in the proceeding section. For qRT-PCR quantification, viral RNA was extracted from rhesus serum using QIAamp Viral RNA Kits (Qiagen) following the manufacturer's instruction. Extracted RNA was eluted in $40 \mu \mathrm{l}$ RNase-free water. qRT-PCR assay was performed as described above. In vitro-transcribed full-length ZIKV RNA was used as a standard for qRT-PCR quantification. The primer/probe set is described in the proceeding section.

Neutralization assay and neurovirulance. All antibody neutralization titers were determined using an mCherry ZIKV as previously reported ${ }^{19}$. The serum dilution that neutralized $50 \%$ of mCherry ZIKV infection $\left(\mathrm{NT}_{50}\right)$ were presented. For measuring neurovirulence, 1-day-old outbred CD-1 mice (Charles River) were injected intracranially with indicated amounts of viruses. The infected mice were monitored for morbidity and mortality as reported previously ${ }^{19}$.

Mosquito infection. For measuring mosquito infection, artificial blood-meal spiked with $10^{6} \mathrm{FFU} / \mathrm{ml}$ of indicated viruses was used to feed A. aegypti mosquitoes (derived from a stock isolated in Galveston, TX, USA), and engorged mosquitoes were incubated at $28{ }^{\circ} \mathrm{C}, 80 \%$ relative humidity on a $12: 12 \mathrm{~h}$ light:dark cycle with ad libitum access to $10 \%$ sucrose. The infection rates were determined at day 7 post feeding as reported previously ${ }^{35}$.

Data analysis. All data were analyzed with GraphPad Prism v7.02 software. Data are expressed as the mean $\pm \mathrm{SD}$. Comparisons of groups were performed using Mann-Whitney test or one-way analysis of variance with a multiple comparisons correction. A $P$-value of $<0.05$ indicates statistical significance.

Data availability. The authors declare that the data supporting the findings of this study are available within the article and its Supplementary Information files, or are available from the authors upon request.

Received: 6 July 2017 Accepted: 20 July 2017

Published online: 22 September 2017 


\section{References}

1. Shan, C. et al. Zika virus: diagnosis, therapeutics, and vaccine. ACS Infect. Dis. 2, 170-172 (2016)

2. Weaver, S. C. et al. Zika virus: history, emergence, biology, and prospects for control. Antiviral Res. 130, 69-80 (2016).

3. Moreira, J., Peixoto, T. M., Siqueira, A. M. \& Lamas, C. C. Sexually acquired Zika virus: a systematic review. Clin. Microbiol. Infect. 23, 296-305 (2017).

4. Cao-Lormeau, V. M. et al. Guillain-barre syndrome outbreak associated with Zika virus infection in French Polynesia: a case-control study. Lancet 387, 1531-1539 (2016)

5. Brasil, P. et al. Zika virus infection in pregnant women in Rio de Janeiro. N. Engl. J. Med. 375, 2321-2334 (2016)

6. Rasmussen, S. A., Jamieson, D. J., Honein, M. A. \& Petersen, L. R. Zika virus and birth defects-reviewing the evidence for causality. N. Engl. J. Med. 374 1981-1987 (2016).

7. Barzon, L. et al. Infection dynamics in a traveller with persistent shedding of Zika virus RNA in semen for six months after returning from Haiti to Italy, January 2016. Euro Surveill. http://dx.doi.org/10.2807/1560-7917. ES.2016.21.32.30316 (2016).

8. Nicastri, E. et al. Persistent detection of Zika virus RNA in semen for six months after symptom onset in a traveller returning from Haiti to Italy, February 2016. Euro Surveill. http://dx.doi.org/10.2807/1560-7917. ES.2016.21.32.30314 (2016).

9. Arsuaga, M., Bujalance, S. G., Diaz-Menendez, M., Vazquez, A. \& Arribas, J. R. Probable sexual transmission of Zika virus from a vasectomised man. Lancet Infect. Dis. 16, 1107 (2016).

10. Russell, K. et al. Male-to-female sexual transmission of Zika virus-United States, January-April 2016. Clin. Infect. Dis. 64, 211-213 (2017).

11. Uraki, R. et al. Zika virus causes testicular atrophy. Sci. Adv. 3, el602899 (2017).

12. Ma, W. et al. Zika virus causes testis damage and leads to male infertility in mice. Cell 167, 1511-1524 (2016).

13. Govero, J. et al. Zika virus infection damages the testes in mice. Nature 540, 438-442 (2016)

14. Larocca, R. A. et al. Vaccine protection against Zika virus from Brazil. Nature 536, 474-478 (2016).

15. Dowd, K. A. et al. Rapid development of a DNA vaccine for Zika virus. Science 354, 237-240 (2016).

16. Abbink, P. et al. Protective efficacy of multiple vaccine platforms against Zika virus challenge in rhesus monkeys. Science 353, 1129-1132 (2016).

17. Richner, J. M. et al. Modified mRNA Vaccines protect against Zika virus infection. Cell 168, 1114-1125 (2017).

18. Pardi, N. et al. Zika virus protection by a single low-dose nucleoside-modified mRNA vaccination. Nature 543, 248-251 (2017).

19. Shan, C. et al. A live-attenuated Zika virus vaccine candidate induces sterilizing immunity in mouse models. Nat. Med. 23, 763-767 (2017).

20. Xie, X. et al. Understanding Zika virus stability and developing a chimeric vaccine through functional analysis. MBio 8 e02134-16 (2017)

21. Betancourt, D., de Queiroz, N. M., Xia, T., Ahn, J. \& Barber, G. N. Cutting edge: innate immune augmenting vesicular stomatitis virus expressing zika virus proteins confers protective immunity. J. Immunol. 198, 3023-3028 (2017).

22. Fernandez, E. \& Diamond, M. S. Vaccination strategies against Zika virus. Curr. Opin. Virol. 23, 59-67 (2017).

23. McArthur, M. A. Zika virus: recent advances towards the development of vaccines and therapeutics. Viruses http://dx.doi.org/10.3390/v90601433 (2017).

24. Richner, J. et al. Vaccine mediated protection against Zika virus induced congenital disease. Cell 170, 273-283 (2017).

25. Griffin, B. D. et al. DNA vaccination protects mice against Zika virus-induced damage to the testes. Nat. Commun. 8, 15743 (2017).

26. Lazear, H. M. et al. A mouse model of Zika virus pathogenesis. Cell Host Microbe 19, 720-730 (2016).

27. Grant, A. et al. Zika virus targets human STAT2 to inhibit type I interferon signaling. Cell Host Microbe 19, 882-890 (2016).

28. Miner, J. J. et al. Zika virus infection during pregnancy in mice causes placental damage and fetal demise. Cell 165, 1081-1091 (2016).

29. Sapparapu, G. et al. Neutralizing human antibodies prevent Zika virus replication and fetal disease in mice. Nature 540, 443-447 (2016).

30. Guerbois, M. et al. Outbreak of zika virus infection, Chiapas State, Mexico, 2015, and first confirmed transmission by Aedes aegypti mosquitoes in the Americas. J. Infect. Dis. 214, 1349-1356 (2016).

31. Ferreira-de-Brito, A. et al. First detection of natural infection of Aedes aegypti with Zika virus in Brazil and throughout South America. Mem. Inst. Oswaldo Cruz 111, 655-658 (2016)
32. Acceptability of cell substrates for production of biologicals. Report of a WHO Study Group on Biologicals. World Health Organ. Tech. Rep. Ser. 747, 1-29 (1987).

33. Ong, E. Z. et al. Preclinical evaluation of VIS513, a therapeutic antibody against dengue virus, in non-human primates. Antiviral Res. 144, 44-47 (2017).

34. Organization, W. H. WHO/UNICEF Zika virus vaccine target product profile: Vaccine to protect congenital Zika syndrome for use during an emergence. http://www.who.int/immunization/research/development/ WHO UNICEF Zikavac TPP Feb2017.pdf?ua=1 (2017).

35. Shan, C. et al. An infectious cDNA clone of Zika virus to study viral virulence, mosquito transmission, and antiviral inhibitors. Cell Host Microbe 19, 891-900 (2016).

36. Hansen, D. A., Esakky, P., Drury, A., Lamb, L. \& Moley, K. H. The aryl hydrocarbon receptor is important for proper seminiferous tubule architecture and sperm development in mice. Biol. Reprod. 90, 8 (2014).

37. Shan, C. et al. A rapid Zika diagnostic assay to measure neutralizing antibodies in patients. EbioMedicine 17, 157-162 (2017).

\section{Acknowledgements}

We thank colleagues at University of Texas Medical Branch for helpful discussions and support during the course of this study. We also thank J.P. Todd and R. Bailer for help with the nonhuman primate studies and sample processing. P.-Y.S. lab was supported by University of Texas Medical Branch (UTMB) startup award, University of Texas STARs Award, CDC grant for the Western Gulf Center of Excellence for Vector-Borne Diseases, Pan American Health Organization grant SCON2016-01353, the Kleberg Foundation award, UTMB CTSA UL1TR-001439 and NIH grant AI127744. K.M.M., W.-P.K., B.S.G. and T.C.P. are funded through intramural funding from the National Institute of Allergy and Infectious Diseases. This research was also partially supported by NIH grant AI120942 to S.C.W. and grants AI073755, AI104972, and AI106695 from the NIH to M.S.D. P.F.C.V. was suported by projects of CAPES (Zika Fast-Track) and CNPq: grants 440405/2016-5, and 303999/2016-0 from the Ministry of Science and Technology of Brazil and by the Ministry of Health.

\section{Author contributions}

C.S., A.E.M., B.W.J., J.R., B.T.D.N., D.B.A.M., and X.X. performed experiments and data analysis. K.M.M., W.-P.K., T.C.P., A.D.B., S.C.W., S.L.R., P.F.C.V., B.S.G., M.S.D., and P.-Y.S. designed the experiments and interpreted the results. C.S., K.M.M., T.C.P., A.D.B., S.C.W., S.L.R., P.F.C.V., B.S.G., M.S.D., and P.-Y.S. wrote the manuscript.

\section{Additional information}

Supplementary Information accompanies this paper at doi:10.1038/s41467-017-00737-8.

Competing interests: C.S. and P.-Y.S. have filed a patent related to the technology presented in this paper. M.S.D. is a consultant for Inbios, Visterra, and Takeda Pharmaceuticals and on the Scientific Advisory Boards of Moderna and OvaGene. The remaining authors declare no competing financial interests.

Reprints and permission information is available online at http://npg.nature.com/ reprintsandpermissions/

Publisher's note: Springer Nature remains neutral with regard to jurisdictional claims in published maps and institutional affiliations.

\footnotetext{
cc) (i) Open Access This article is licensed under a Creative Commons Attribution 4.0 International License, which permits use, sharing adaptation, distribution and reproduction in any medium or format, as long as you give appropriate credit to the original author(s) and the source, provide a link to the Creative Commons license, and indicate if changes were made. The images or other third party material in this article are included in the article's Creative Commons license, unless indicated otherwise in a credit line to the material. If material is not included in the article's Creative Commons license and your intended use is not permitted by statutory regulation or exceeds the permitted use, you will need to obtain permission directly from the copyright holder. To view a copy of this license, visit http://creativecommons.org/ licenses/by/4.0/.
}

(c) The Author(s) 2017 